\title{
Gene fusion detection in formalin-fixed paraffin-embedded benign fibrous histiocytomas using fluorescence in situ hybridization and RNA sequencing
}

\author{
Charles Walther', Jakob Hofvander', Jenny Nilsson', Linda Magnusson', Henryk A Domanski², David Gisselsson ,2, \\ Johnbosco Tayebwa', Leona A Doyle ${ }^{3}$, Christopher DM Fletcher ${ }^{3}$ and Fredrik Mertens ${ }^{1}$
}

Benign fibrous histiocytomas (FH) can be subdivided into several morphological and clinical subgroups. Recently, gene fusions involving either one of two protein kinase $\mathrm{C}$ genes (PRKCB and PRKCD) or the ALK gene were described in FH. We here wanted to evaluate the frequency of $P R K C B$ and $P R K C D$ gene fusions in $\mathrm{FH}$. Using interphase fluorescence in situ hybridization on sections from formalin-fixed paraffin-embedded (FFPE) tumors, 36 cases could be analyzed. PRKCB or $P R K C D$ rearrangements were seen in five tumors: $1 / 7$ regular, $0 / 3$ aneurysmal, $0 / 6$ cellular, 2/7 epithelioid, 0/1 atypical, 2/10 deep, and $0 / 2$ metastatic lesions. We also evaluated the status of the $A L K$ gene in selected cases, finding rearrangements in 3/7 epithelioid and 0/1 atypical lesions. To assess the gene fusion status of FH further, deep sequencing of RNA (RNASeq) was performed on FFPE tissue from eight cases with unknown gene fusion status, as well as on two FH and six soft tissue sarcomas with known gene fusions; of the latter eight positive controls, the expected fusion transcript was found in all but one, while $2 / 8 \mathrm{FH}$ with unknown genetic status showed fusion transcripts, including a novel KIRREL/PRKCA chimera. Thus, also a third member of the PRKC family is involved in $\mathrm{FH}$ tumorigenesis. We conclude that gene fusions involving PRKC genes occur in several morphological (regular, cellular, aneurysmal, epithelioid) and clinical (cutaneous, deep) subsets of $\mathrm{FH}$, but they seem to account for only a minority of the cases. In epithelioid lesions, however, rearrangements of PRKC or ALK were seen, as mutually exclusive events, in the majority (5/7) of cases. Finally, the study also shows that RNA-Seq is a promising tool for identifying gene fusions in FFPE tissues.

Laboratory Investigation (2015) 95, 1071-1076; doi:10.1038/labinvest.2015.83; published online 29 June 2015

Benign fibrous histiocytoma (FH) can be subdivided into several morphological and clinical subgroups. Morphological variants include cellular, aneurysmal, epithelioid, and atypical types, and clinical manifestations range from benign tumors of the skin, deep soft tissues or skeleton to, rarely, metastasizing tumors. ${ }^{1-3}$ We recently showed that both cutaneous and deep FH may carry specific gene fusions, in which a membereither $P R K C B$ and $P R K C D$ - of the gene family encoding protein kinase $\mathrm{C}$ (PRKC) is juxtaposed with a gene encoding a membrane-associated protein. ${ }^{4}$ The pathogenetic mechanism was assumed to involve the uncoupling of the carboxy-terminal kinase domain of the PRKC protein from its regulatory domain, and its ectopic localization through fusion with the amino-terminal part of a membrane-associated protein. It has also recently been shown that some cases of epithelioid, and possibly also atypical, FH may harbor fusions activating the
ALK protein. 5,6 The connection between $A L K$ rearrangements and FH was further evaluated and strengthened by Doyle et al., showing that $A L K$ fusions were restricted to the epithelioid subtype. $^{7}$

To study the frequency and distribution of $P R K C B$ and $P R K C D$ fusions in $\mathrm{FH}$, we used interphase fluorescence in situ hybridization (FISH) on a series of tumors that represented different morphological and clinical subsets; the results of that study prompted transcriptome sequencing (RNA-Seq) of RNA from formalin-fixed paraffin-embedded (FFPE) tissue from a second set of $\mathrm{FH}$.

\section{MATERIALS AND METHODS Patients and Tumors}

For the interphase FISH studies, 66 cases of FH were retrieved from the consultation files of one of the authors (CDM

${ }^{1}$ Department of Clinical Genetics, University and Regional Laboratories, Skåne University Hospital, Lund University, Lund, Sweden; ${ }^{2}$ Department of Pathology, University and Regional Laboratories, Skåne University Hospital, Lund, Sweden and ³Department of Pathology, Brigham and Women's Hospital, Harvard Medical School, Boston, MA, USA Correspondence: Dr C Walther, MD, Department of Clinical Genetics, University and Regional Laboratories, Skåne University Hospital, Lund 221 85, Sweden.

E-mail: charles.walther@med.lu.se

Received 9 March 2015; revised 4 May 2015; accepted 13 May 2015 
Fletcher). The cases were selected to represent different clinical and morphological aspects of FH: 10 regular, 10 cellular, 10 aneurysmal, 10 atypical, 10 epithelioid, 11 deep, and 5 metastatic lesions; no prior genetic information was available on these tumors, except for two epithelioid FH that had been analyzed with regard to $A L K$ gene rearrangements. The diagnostic criteria have been outlined before. ${ }^{1,8-13}$ Two cases of regular $\mathrm{FH}$ with known fusions involving $P R K C B$ and $P R K C D$ (previously published as Cases 5 and 6 in $^{4}$ ), respectively, were used as positive controls. All seven epithelioid FH that could be analyzed by FISH were also analyzed with regard to expression of the ALK protein, as described. ${ }^{7}$

For the RNA-Seq study, another set of six recently (20112013) diagnosed cutaneous regular FH was chosen. In addition, we included two cytogenetically analyzed $\mathrm{FH}$ from which no frozen tissue was available: one cellular/aneurysmal FH from 1990 (Case 7 in Plaszczyca et al. ${ }^{4}$ and Case Lu 317 in Vanni et al. ${ }^{14}$ ) with an unbalanced $\mathrm{t}(3 ; 12)$ and one cellular FH from 2010 with a balanced $\mathrm{t}(1 ; 16)(\mathrm{p} 36 ; \mathrm{p} 11)$, strongly suggestive of a $P D P N / P R K C B$ fusion. As positive controls, we selected two FH from 2009 with PDPN/PRKCB fusions (Cases 2 and 5 in Plaszczyca et al. ${ }^{4}$ ), four myxoid liposarcomas (MLS) from 2011 or 2014 with FUS/DDIT3 fusions, one synovial sarcoma from 2013 with an SS18/SSX2 fusion, and one low-grade fibromyxoid sarcoma from 2013 with an FUS/CREB3L2 fusion; the fusion gene status of the positive controls had been established by G-banding, FISH, and/or RT-PCR (data not shown). Thus, the RNA-Seq study included eight FH with unknown fusion gene status and eight soft tissue tumors, including two cases of $\mathrm{FH}$, with known gene fusions.

\section{FISH}

Interphase FISH for the detection of rearrangements of $P R K C B$ and $P R K C D$, mapping to chromosome bands $16 \mathrm{p} 12$ and $3 \mathrm{p} 21$, respectively, was performed on $4 \mu \mathrm{m}$ FFPE tumor sections. The bacterial artificial chromosome (BAC) probes were obtained from the BACPAC Resource Center (http://bacpac.chori.org; Supplementary Table 1). Clone preparation, hybridization, and analysis were performed as described. ${ }^{15,16}$ On average 113 (range $=83-176$ ) nuclei from different areas were evaluated per probe set in each case. Nuclei displaying separate green and red signals or a single $3^{\prime}$ signal were scored as positive. In seven epithelioid and one atypical $\mathrm{FH}$, the status of the $A L K$ gene was also investigated, using a commercial double color, break-apart probe (LSI ALK, Vysis, Abbott Molecular, IL, USA); in each case, 95-112 nuclei were scored. The status of the PRKCA gene was evaluated in Case 39, using probes described in Supplementary Table 1; 93 nuclei were scored.

\section{RNA-Seq and RT-PCR}

For RNA extraction, tumor blocks from each of the 16 cases (Cases 37-44 and PC3-10) were retrieved and tumorrepresentative areas were selected. Then, $10 \mu \mathrm{m}$ sections were cut from each tumor $(n=3-38$, depending on the size of the selected areas; the aim was to achieve a total of $450 \mathrm{~mm}^{2}$ of tumor tissue from each case). The first two sections were discarded and the following sections were immediately put in Deparaffinization Solution (Qiagen, Valencia, CA, USA) followed by RNA extraction with Qiagen's RNeasy FFPE Kit, according to the manufacturer's recommendations. The quality and quantity of the RNA, with a particular focus on the fraction of RNA fragments $>200 \mathrm{nt}$ (the $\mathrm{DV}_{200}$ ), were measured using an Agilent 2100 Bioanalyzer (Agilent Technologies, Palo Alto, CA, USA). mRNA libraries were prepared from 20 to $50 \mathrm{ng}$, depending on the $\mathrm{DV}_{200}$ value, of the extracted RNA using the capturing chemistry of the TruSeq RNA Access Library Prep Kit (Illumina, San Diego, CA, USA), according to the manufacturer's recommendations. The quality of the mRNA library was measured using an Agilent 2100 Bioanalyzer (Agilent Technologies) and the concentration was measured on a Qubit Fluorometer using the Qubit RNA HS Assay kit (Life Technologies, Rockville, MD, USA). With a loading concentration of $1.8 \mathrm{pM}$, pairedend $2 \times 81$ nt reads were generated in-house from the mRNA libraries using Illumina's High Output Kit (150 cycles) on a NextSeq 500 (Illumina). To identify candidate fusion transcripts from the sequence data, analyses were performed on fastq files using ChimeraScan version 0.4.5 and TopHat version 2.0.7, ${ }^{17,18}$ as decribed. ${ }^{19,20}$ The GRCh37/hg19 build was used as the human reference genome.

RT-PCR was performed in Case 39, using primers specific for KIRREL (forward, 5'-GACCGGGAGGATGACACCG-3') and PRKCA (reverse, 5'-GGGACTGATGACTTTGTT GCC-3'). RT-PCR and sequencing were performed as described. ${ }^{16}$

\section{RESULTS \\ FISH}

For technical reasons, interphase FISH for $P R K C B$ and/or $P R K C D$ rearrangements was possible to perform in only 36 of the selected FH cases (cases 1-36) and in two positive controls (PC1-2; Table 1); the remaining samples did not yield signals of sufficient quality. The distribution of positive nuclei differed between the two probe sets: the mean numbers of positive nuclei for $P R K C B$ and $P R K C D$ rearrangements were $8.4 \%$ and $15.5 \%$, with standard deviations (s.d.) $7.1 \%$ and $11.3 \%$, respectively. Cutoff values for scoring cases as positive were chosen at $>23 \%$ for $P R K C B$ and $>38 \%$ for $P R K C D$ (i.e., mean +2 s.d.). The cutoff for the commercial ALK probe was set at $>15 \%$. Using these criteria, the two positive controls, i.e., tumors in which we previously had identified fusions using RT-PCR on frozen tissue, ${ }^{4}$ were positive for the expected $P R K C$ gene and negative for the other (Table 1). $P R K C B$ was scored as positive in $3 / 34$ cases and $P R K C D$ was positive in $2 / 31$ cases. The $P R K C$-positive $\mathrm{FH}$ cases were distributed as follows: $1 / 7$ regular, $0 / 3$ aneurysmal, $0 / 6$ cellular, 2/7 epithelioid, $0 / 1$ atypical, 2/10 deep, $0 / 2$ metastatic (Table 1 ). The two epithelioid FH that had $P R K C B$ rearrangements at 
Table 1 Results of FISH analyses on FFPE sections from benign fibrous histiocytomas ${ }^{\mathrm{a}}$

\begin{tabular}{|c|c|c|c|c|}
\hline Case No. & Subtype & PRKCB (\%) & PRKCD (\%) & $A L K(\%)$ \\
\hline PC1 & PRKCD + & Neg (2) & Pos (44) & ND \\
\hline PC2 & PRKCB+ & Pos (51) & Neg (14) & ND \\
\hline 1 & Regular & Neg (9) & Pos (47) & ND \\
\hline 2 & Regular & Neg (16) & Neg (12) & ND \\
\hline 3 & Regular & Neg (8) & Neg (15) & ND \\
\hline 4 & Regular & Neg (18) & Neg (14) & ND \\
\hline 5 & Regular & Neg (1) & Neg (6) & ND \\
\hline 6 & Regular & Neg (8) & Neg (10) & ND \\
\hline 7 & Regular & Neg (6) & Neg (12) & ND \\
\hline 8 & Aneurysmal & Neg (7) & Neg (33) & ND \\
\hline 9 & Aneurysmal & Neg (1) & Neg (11) & ND \\
\hline 10 & Aneurysmal & Neg (6) & Neg (14) & ND \\
\hline 11 & Cellular & Neg (6) & Neg (17) & ND \\
\hline 12 & Cellular & Neg (17) & Neg (15) & ND \\
\hline 13 & Cellular & Neg (9) & ND & ND \\
\hline 14 & Cellular & Neg (10) & ND & ND \\
\hline 15 & Cellular & Neg (11) & Neg (7) & ND \\
\hline 16 & Cellular & ND & Neg (9) & ND \\
\hline 17 & Epithelioid & Neg (3) & Neg (10) & $\operatorname{Pos}(\mathbf{5 8})^{\mathrm{b}, \mathrm{c}}$ \\
\hline 18 & Epithelioid & ND & Neg (31) & $\operatorname{Pos}(66)^{c}$ \\
\hline 19 & Epithelioid & Pos (25) & Neg (17) & Neg $(11)^{b, d}$ \\
\hline 20 & Epithelioid & Neg (11) & Neg (8) & $\operatorname{Pos}(38)^{c}$ \\
\hline 21 & Epithelioid & Neg (9) & ND & $\operatorname{Neg}(6)^{c}$ \\
\hline 22 & Epithelioid & Pos $(23)$ & ND & $\operatorname{Neg}(1)^{d}$ \\
\hline 23 & Epithelioid & Neg (8) & ND & $\operatorname{Neg}(11)^{c}$ \\
\hline 24 & Atypical & Neg (7) & Neg (17) & Neg (1) \\
\hline 25 & Deep & Neg (0) & Neg (15) & ND \\
\hline 26 & Deep & Neg (2) & Neg (12) & ND \\
\hline 27 & Deep & Neg (3) & Neg (17) & ND \\
\hline 28 & Deep & Neg (3) & Neg (14) & ND \\
\hline 29 & Deep & Neg (9) & Neg (12) & ND \\
\hline 30 & Deep & Neg (7) & Pos (56) & ND \\
\hline 31 & Deep & Neg (1) & Neg (1) & ND \\
\hline 32 & Deep & Neg (3) & Neg (25) & ND \\
\hline 33 & Deep & Neg (1) & Neg (8) & ND \\
\hline 34 & Deep & Pos (30) & Neg (24) & ND \\
\hline 35 & Metastatic & ND & Neg (5) & ND \\
\hline 36 & Metastatic & Neg (4) & Neg (14) & ND \\
\hline
\end{tabular}

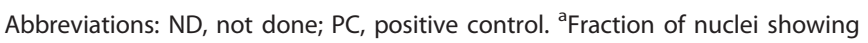
split signals is indicated in the parentheses. Cases with frequencies exceeding cutoff values are highlighted in bold. ${ }^{b}$ Previously also analyzed by Doyle


tochemistry. ${ }^{\mathrm{d}}$ Negative for ALK expression at immunohistochemistry.
FISH analysis were negative for ALK protein expression (Table 1).

The status of the $A L K$ gene had previously been studied in two cases of epithelioid $\mathrm{FH}$, one being negative and one positive. ${ }^{7}$ Here, these results could be repeated, and another two cases of epithelioid $\mathrm{FH}$, both negative for PRKC rearrangements, were scored as positive. Thus, 3/7 epithelioid and $0 / 1$ atypical $\mathrm{FH}$ were ALK positive (Table 1 ).

The status of the PRKCA gene was investigated by interphase FISH on cut sections from Case 39, in which a KIRREL/PRKCA fusion transcript was found by both RNASeq and RT-PCR (described below). The FISH analysis disclosed a split signal, consistent with translocation, in $42 \%$ of the nuclei.

\section{RNA-Seq and RT-PCR}

RNA of sufficient quantity (range $=2.9-43.4 \mu \mathrm{g}$, median $=9.7$ $\mu \mathrm{g})$ and quality $\left(\mathrm{DV}_{200}\right.$ value: range $=45-75 \%$, mean $\left.=60 \%\right)$ could be extracted from all 16 tumors. The number of reads obtained per case ranged from 14.5 to $64.4 \times 10^{6}$, median $=$ $25.2 \times 10^{6}$ (Table 2). The two software used in this study identified numerous potential fusion transcripts in all cases (Supplementary Tables 2 and 3), the vast majority of which could be discarded as read-through transcripts or technical artifacts. A single, biologically relevant fusion transcript, all of which were identified by ChimeraScan, was found in nine cases; five of these were found also with TopHat (Table 2). The expected fusion transcripts were found in 5/6 sarcomas with known gene fusions (three MLS with the FUS/DDIT3 fusion, one synovial sarcoma with the SS18/SSX2 fusion, and one low-grade fibromyxoid sarcoma with the FUS/CREB3L2 fusion); one MLS did not display the expected FUS/DDIT3 fusion by any of the two software. Also the two FH with known PDPN/PRKCB fusions were identified at RNA-Seq of FFPE tissue. Thus, $7 / 8$ positive controls were correctly classified. In addition, one FH with a known $\mathrm{t}(1 ; 16)$ showed a $P D P N / P R K C B$ fusion, in agreement with the karyotype, whereas one $\mathrm{FH}$ with an unbalanced $\mathrm{t}(3 ; 12)$ was negative. Of the remaining six FH (Cases 37-44), five did not display any relevant fusion transcript, whereas one showed a novel KIRREL/PRKCA fusion (Table 2). This fusion was verified by RT-PCR, revealing an in-frame fusion of exon 13 (nt 158,093,762) of KIRREL with exon 9 (nt 66,732,687) of PRKCA (Positions according to GRCh38; Figure 1).

\section{DISCUSSION}

We recently showed that gene fusions involving the $P R K C B$ and $P R K C D$ genes are recurrent in FH. ${ }^{4}$ However, that study was biased in the sense that it only included tumors that had clonal chromosomal rearrangements at G-banding analysis, potentially enriching certain genetic subgroups with a high proliferative capacity in vitro. Furthermore, only four cases in that study could be analyzed by molecular and/or FISH techniques. We thus wanted to explore a larger series of $\mathrm{BFH}$, with tumors representing all known morphologic subtypes. 
Table 2 Benign fibrous histiocytomas and soft tissue sarcomas used for RNA-Seq on FFPE tissue

\begin{tabular}{|c|c|c|c|c|c|c|c|}
\hline Case no. & Diagnosis & Year & $\begin{array}{l}\text { Known/predicted } \\
\text { fusion status }\end{array}$ & $\begin{array}{c}\text { Percentage } \\
\text { fragments }>200 \mathrm{nt}(\%)\end{array}$ & Read count & TopHat & $\begin{array}{l}\text { Chimerascan (total No of } \\
\text { fragments supporting, spanning fragments) }\end{array}$ \\
\hline PC3 & $\mathrm{RFH}$ & 2009 & PDPN/PRKCB & 53 & 20950432 & PDPN/PRKCB & PDPN/PRKCB $(51,21)$ \\
\hline PC4 & $\mathrm{RFH}$ & 2009 & PDPN/PRKCB & 62 & 16872619 & PDPN/PRKCB & PDPN/PRKCB $(44,14)$ \\
\hline 37 & $\mathrm{CFH}$ & 2010 & PDPN/PRKCB? & 66 & 33084533 & PDPN/PRKCB & PDPN/PRKCB $(119,47)$ \\
\hline 40 & $\mathrm{RFH}$ & 2011 & Unknown & 56 & 17936136 & Neg & Neg \\
\hline 41 & $\mathrm{RFH}$ & 2012 & Unknown & 58 & 18311561 & Neg & Neg \\
\hline 42 & $\mathrm{RFH}$ & 2012 & Unknown & 45 & 33233957 & Neg & Neg \\
\hline 43 & $\mathrm{RFH}$ & 2012 & Unknown & 72 & 40665408 & Neg & Neg \\
\hline PC7 & MLS & 2014 & FUS/DDIT3 & 75 & 23576431 & Neg & FUS/DDIT3 $(14,0)$ \\
\hline PC8 & MLS & 2014 & FUS/DDIT3 & 54 & 39879954 & Neg & FUS/DDIT3 $(16,1)$ \\
\hline PC9 & MLS & 2011 & FUS/DDIT3 & 56 & 14764666 & Neg & Neg \\
\hline PC10 & MLS & 2014 & FUS/DDIT3 & 55 & 28966064 & Neg & FUS/DDIT3 $(12,0)$ \\
\hline
\end{tabular}

Abbreviations: CAFH, cellular/aneurysmal fibrous histiocytoma; CFH, cellular fibrous histiocytoma; LGFMS, low-grade fibromyxoid sarcoma; MLS, myxoid liposarcoma; PC, positive control; RFH, regular fibrous histiocytoma; Syn sarc, synovial sarcoma.

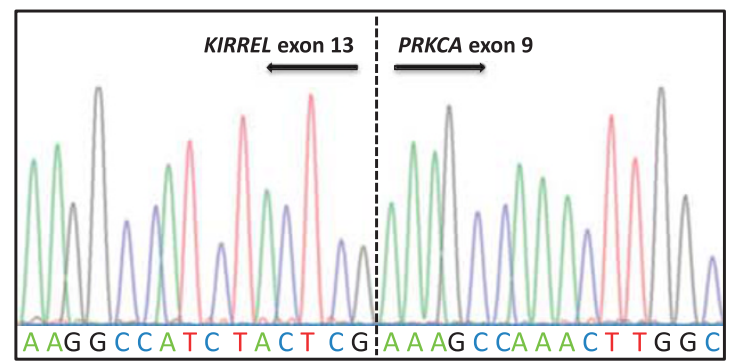

Figure 1 Chromatogram depicting the fusion between KIRREL and PRKCA in a benign fibrous histiocytoma.

For that purpose, unstained sections from 66 cases of $\mathrm{BFH}$ were retrieved and studied with regard to $P R K C B$ and $P R K C D$ rearrangements using interphase FISH. However, this analysis turned out to be unusually challenging in the sense that it was very difficult to obtain signals of sufficient quality. Because FISH analyses with commercial probes for the $A L K$ gene have been successfully performed on FFPE sections from $\mathrm{FH},{ }^{7}$ we assume that the technical problems were due to the probe sets we used. However, also the age of the samples could possibly have affected the results. Nevertheless, reliable results could be obtained in 36 of the cases; 5 of them were interpreted to have a $P R K C B$ or a $P R K C D$ rearrangement (Table 1). As this frequency $(5 / 36,14 \%)$ of $P R K C B / P R K C D$-positive cases was considerably lower than in our initial study and because the positive cases were found among several of the different morphologic/clinical subsets_-regular, epithelioid, and deep lesions-we wanted to use another technique to investigate whether the discrepancy was due to poor sensitivity of FISH. With no further material being available from the intial cohort, a second set of recently diagnosed regular $\mathrm{FH}$ was retrieved for RNA extraction and RNA-Seq. This approach turned out to be technically highly successful, in terms of RNA quantity and quality as well as sequencing results (Table 2). With only $1 / 8$ positive controls being negative for the expected gene fusion, it seems reasonable to assume that the results on the FH with unknown genetic status were fairly accurate; the negative case (PC9) was the positive control yielding the lowest number of reads, possibly explaining the negative results (Table 2).

The RNA-Seq analysis identified one cellular FH with the $P D P N / P R K C B$ fusion that has been previously detected in $\mathrm{FH}^{4}{ }^{4}$ In addition, 1/6 regular $\mathrm{FH}$ displayed a novel fusion between KIRREL and PRKCA, mapping to $1 \mathrm{q} 23$ and 17q24, respectively. Although KIRREL has not been implicated in gene fusions before, Bridge and co-workers recently described a recurrent SLC44A1/PRKCA fusion in papillary glioneural tumor, with the same breakpoint in PRKCA as in the FH of the present study. ${ }^{21}$ Further arguments for a pathogenetic significance of the KIRREL/PRKCA fusion in the context of $\mathrm{FH}$ is that KIRREL, like the previously identified aminoterminal partners PDPN, CD63, and LAMTOR1, is a membrane-associated protein, retaining its membranebinding part in the chimeric protein. ${ }^{4}$ 
We can thus conclude that $P R K C$ gene rearrangements are indeed recurrent in $\mathrm{FH}$ and that, based on the present and our previous study, they at least occur in the regular, cellular, aneurysmal, and epithelioid subtypes, and that they may be found in both cutaneous and deep lesions; more cases of atypical and metastatic FH need to be analyzed before it can be excluded that also these entities may harbor PRKC fusions. The overall frequency of $P R K C$-positive FHs can only be roughly estimated. First, interphase FISH was only performed for two members of the PRKC gene family ( $P R K C B$ and $P R K C D$ ) but we now know from the RNA-Seq study that at least the alpha variant (PRKCA) can also be involved in fusions. Second, results of interphase FISH on FFPE sections are somewhat difficult to standardize, as the samples may vary significantly in technical quality. If we had lowered the cutoff values to 1 s.d. above the mean frequencies of split signals, four more cases would have been scored as positive for PRKC rearrangements, whereas an increase of the cutoff to 3 s.d. above mean frequencies would have turned two PRKCDpositive cases, including the positive control, and two $P R K C B$-positive cases into negative ones (Table 1); unfortunately, no tissue blocks for RNA extraction were available from the cases that were used for FISH. Taking the methodological shortcomings mentioned above into account, our results from FISH and RNA-Seq suggest that only some $15-25 \%$ of $\mathrm{FH}$ are positive for PRKC gene fusions. Thus, there must exist alternative pathogenetic pathways in $\mathrm{FH}$. Indeed, it was recently shown by both FISH and RNA-Seq that epithelioid FH carry fusions involving the ALK geneVCL/ALK and SQSTM1/ALK, respectively. ${ }^{6}$ This finding was further expanded by Doyle and co-workers who analyzed 33 epithelioid FH by immunohistochemistry (IHC) - 29/33 cases were ALK-positive, and 14/14 of these turned out to be ALKrearranged also at FISH analysis; other subtypes of FH were IHC-negative for ALK. ${ }^{7}$ Interestingly, epithelioid $\mathrm{FH}$ was the subtype most often (2/7) showing a PRKC gene rearrangement in our FISH study (Table 1), suggesting that the vast majority of these lesions harbor either an $A L K$ fusion or a $P R K C$ fusion. The conclusion that $A L K$ and $P R K C$ activation constitute alternative, and not overlapping, pathogenetic pathways in epithelioid $\mathrm{FH}$ was supported by the finding that the two cases with $P R K C B$ rearrangement were negative for ALK expression, whereas the other five cases were positive (Table 1); there were no obvious morphological or clinical differences between PRKC- and ALK-positive epithelioid FH, but it should be kept in mind that very few cases were analyzed. Also two tumors diagnosed as atypical $\mathrm{FH}$ were recently reported to have ALK rearrangements, as shown by FISH or IHC, $^{5}$ but, in our opinion, the morphologic description and images would fit better with epithelioid FH. The single atypical FH (Case 24) that we could analyze by FISH was negative for $P R K C B, P R K C D$, and ALK rearrangements (Table 1).

The RNA-Seq part of our study adds to the still small but growing number of reports showing that the NGS technologies are applicable to RNA extracted from FFPE tissues. ${ }^{22-26}$ Furthermore, RNA-Seq on FFPE tissue has only rarely been used to detect gene fusions. ${ }^{6,23,27}$ The study of Sweeney and co-workers is of particular interest in the present context, as they also investigated sarcomas (three synovial sarcomas, three MLS, two Ewing sarcomas, and one clear cell sarcoma), used Illumina equipment and kits, and had results similar to ours; ${ }^{23}$ all their cases showed the expected gene fusions, although some of the samples had to be re-analyzed at greater depth or after depletion of ribosomal RNA before obtaining positive results. Some of the methodological differences between their and our study deserve further comments. First, Sweeney and co-workers read longer sequences than we did $(2 \times 150 \mathrm{nt}$, compared with $2 \times 81 \mathrm{nt}$ in our study). In principle, longer reads should have a greater chance of covering the fusion breakpoints (spanning reads) and while all their cases had fusion spanning reads, three of our positive controls did not (Table 2). As shown in our study, however, flanking reads can also accurately identify gene fusions. Second, none of the samples analyzed by Sweeney and co-workers had been archived for more than 6 months, whereas our oldest positive sample had been archived for 5 years. Most likely, even older samples can be successfully studied with regard to gene fusion status. Hedegaard et al. performed a comprehensive analysis of RNA-Seq data on both FFPE and fresh frozen samples from various carcinomas that had been stored up to 244 months; the two methods showed very good correspondence in terms of up- and downregulated genes. ${ }^{24}$ Third, Sweeney and coworkers selected reads aligning only to genes known to be involved in sarcoma-associated fusions, a so-called sarcomatome, whereas we extracted all potential fusion genes. Our strategy did not result in any potential false positives, and did not significantly increase the time needed to identify the fusion of interest. Most importantly, our unbiased approach allowed us to find a novel fusion transcript, where none of the two genes had been implicated in soft tissue tumors before. In summary, we find the results of our RNA-Seq study very promising and believe that FFPE samples can be successfully used for gene fusion detection.

Supplementary Information accompanies the paper on the Laboratory Investigation website (http://www.laboratoryinvestigation.org)

\section{ACKNOWLEDGMENTS}

We thank Caroline Jansson for technical assistance with the cutting of FFPE sections for the RNA-Seq studies and Henrik Lilljebjörn for valuable discussions. This study was supported by the Swedish Cancer Society and the Gunnar Nilsson Cancer Foundation.

\section{DISCLOSURE/CONFLICT OF INTEREST}

The authors declare no conflict of interest.

1. Gleason BC, Fletcher CD. Deep "benign" fibrous histiocytoma: clinicopathologic analysis of 69 cases of a rare tumor indicating occasional metastatic potential. Am J Surg Pathol 2008;32:354-362. 
2. Luzar B, Calonje E. Cutaneous fibrohistiocytic tumours-an update. Histopathology 2010;56:148-165.

3. Nielsen GP, Kyriakos M. Non-ossifying fibroma/benign fibrous histiocytoma of bone. In: Fletcher CD, Bridge JA, Hogendoorn PCW, Mertens F (eds). World Health Organization Classification of Tumours. Pathology and Genetics of Tumours of Soft Tissue and Bone. IARC Press: Lyon, 2013, pp 302-304.

4. Plaszczyca A, Nilsson J, Magnusson L et al. Fusions involving protein kinase $C$ and membrane-associated proteins in benign fibrous histiocytoma. Int J Biochem Cell Biol. 2014;53:475-481.

5. Szablewski V, Laurent-Roussel S, Rethers L et al. Atypical fibrous histiocytoma of the skin with CD30 and p80/ALK1 positivity and ALK gene rearrangement. J Cutan Pathol 2014;41:715-719.

6. Jedrych J, Nikiforova M, Kennedy TF et al. Epithelioid cell histiocytoma of the skin with clonal $A L K$ gene rearrangement resulting in VCL-ALK and SQSTM1-ALK gene fusions. Br J Dermatol 2015;172:1427-1429.

7. Doyle LA, Marino-Enriquez A, Fletcher CD et al. ALK rearrangement and over-expression in epithelioid fibrous histiocytoma. Mod Pathol, in press.

8. Calonje E, Mentzel T, Fletcher CD. Cellular benign fibrous histiocytoma Clinicopathologic analysis of a distinctive variant of cutaneous fibrous histiocytoma with frequent recurrence. Am J Surg Pathol 1994;18: 668-676.

9. Singh Gomez C, Calonje E, Fletcher CD. Epithelioid benign fibrous histiocytoma of skin: clinic-pathological analysis of 20 cases of a poorly known variant. Histopathology 1994;24:123-129.

10. Calonje E, Fletcher CD. Aneurysmal benign fibrous histiocytoma clinicopathological analysis of 40 cases of a tumour frequently misdiagnosed as a vascular neoplasm. Histopathology 1995;26:323-331.

11. Kaddu S, McMenamin M, Fletcher CD. Atypical fibrous histiocytoma of the skin. Clinicopathologic analysis of 59 cases with evidence of infrequent metastasis. Am J Surg Pathol 2002;26:35-46.

12. Doyle LA, Fletcher CD. EMA positivity in epithelioid fibrous histiocytoma: a potential diagnostic pitfall. J Cutan Pathol 2011;38: 697-703.

13. Doyle LA, Fletcher CD. Metastasizing "benign" cutaneous fibrous histiocytoma. A clinicopathologic analysis of 16 cases. Am J Surg Pathol 2013;37:484-495.

14. Vanni R, Fletcher CD, Sciot R et al. Cytogenetic evidence of clonality in cutaneous benign fibrous histiocytomas: A report of the CHAMP study group. Histopathology 2000;37:212-217.
15. Arbajian E, Puls F, Magnusson L et al. Recurrent EWSR1-CREB3L1 gene fusions in sclerosing epithelioid fibrosarcoma. Am J Surg Pathol 2014:38:801-808.

16. Walther C, Tayebwa J, Lilljebjörn $\mathrm{H}$ et al. A novel SERPINE1-FOSB fusion results in transcriptional up-regulation of FOSB in pseudomyogenic hemangioendothelioma. J Pathol 2014;232:534-540.

17. Iyer MK, Chinnaiyan AM, Maher CA. ChimeraScan: a tool for identifying chimeric transcription in sequencing data. Bioinformatics 2011;27: 2903-2904.

18. Kim D, Pertea G, Trapnell C et al. TopHat2: accurate alignment of transcriptomes in the presence of insertions, deletions and gene fusions. Genome Biol 2013;14:R36.

19. Hofvander J, Tayebwa J, Nilsson J et al. Recurrent PRDM10 gene fusions in undifferentiated pleomorphic sarcoma. Clin Cancer Res 2015;21: 864-869.

20. Hofvander J, Tayebwa J, Nilsson J et al. RNA sequencing of sarcomas with simple karyotypes: identification and enrichment of fusion transcripts. Lab Invest 2015;95:603-609.

21. Bridge JA, Liu X-Q, Sumegi J et al. Identification of a novel, recurrent SLC44A1-PRKCA fusion in papillary glioneuronal tumor. Brain Pathol 2013;23:121-128.

22. Adiconis $X$, Borges-Rivera D, Satija $R$ et al. Comparative analysis of RNA sequencing methods for degraded or low-input samples. Nat Methods 2013;10:623-629.

23. Sweeney RT, Zhang B, Zhu SX et al. Desktop transcriptome sequencing from archival tissue to identify clinically relevant translocations. Am J Surg Pathol 2013;37:796-803.

24. Hedegaard J, Thorsen K, Lund MK et al. Next-generation sequencing of RNA and DNA isolated from paired fresh-frozen and formalin-fixed paraffin-embedded samples of human cancer and normal tissue. PLoS ONE 2014;9:e98187.

25. Li P, Conley A, Zhang H, Kim HL. Whole-transcriptome profiling of formalin-fixed, paraffin-embedded renal cell carcinoma by RNA-seq. BMC Genomics 2014;15:1087.

26. Zhao W, He X, Hoadley KA et al. Comparison of RNA-Seq by poly (A) capture, ribosomal RNA depletion, and DNA microarray for expression profiling. BMC Genomics 2014;15:419.

27. Ma $Y$, Ambannavar R, Stephans J et al. Fusion transcript discovery in formalin-fixed paraffin-embedded human breast cancer tissues reveals a link to tumor progression. PLoS ONE 2014;9:e94202. 